\title{
Lone Spin Remains Shielded Despite Superconductivity
}

\author{
Researchers explore the question of whether a Kondo cloud-a \\ phenomenon common in conventional metals-can also occur in \\ superconductors.
}

By Rachel Berkowitz

n many metals, as the temperature drops, so does electrical
resistance-but only until some threshold temperature,
at which point resistance begins to increase. That puzzle can be explained by the presence of magnetic atoms in the metal. Unpaired electron spins in these impurities couple collectively with all of the metal's free-flowing electrons to form an antiferromagnetic region called a Kondo cloud. Electrons within the cloud are impeded, increasing the material's resistance, while the atoms' unpaired spins are screened from their surroundings. Does the same phenomenon occur in superconductors, whose electron-pairing mechanism and many-body states are different? Paşcu Moca of the University of Oradea, Romania, in collaboration with Gergely Zaránd's group at the Budapest University of Technology and Economics, Hungary, now provide evidence that the Kondo cloud may indeed survive in a superconductor [1].

Using numerical and analytical models, the researchers found

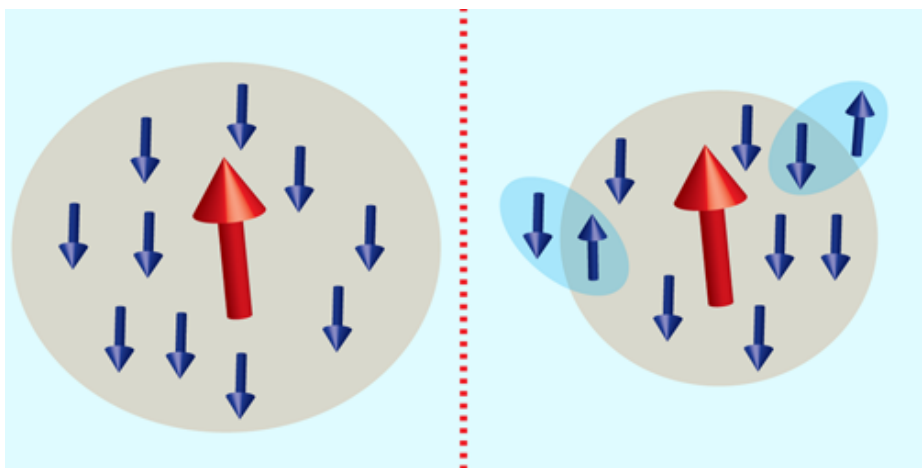

that, for sufficiently strong exchange interactions between the atomic impurity and the superconducting host, the impurity formed a many-body singlet state, meaning that electrons formed a Kondo cloud and paired to the impurity's spin as they would in a conventional metal. For weaker exchange interactions, the impurity's spin remained unpaired. But even in that so-called doublet state, a weak screening cloud persisted. Moca and his colleagues determined that the strength of this screening cloud decayed exponentially in space, with its overall size depending on the superconductor's band gap.

The finding that Kondo clouds appear in superconductors as well as conventional metals has implications for the behavior of quantum states in magnetic systems coupled to superconductors. Such systems are under investigation as a possible information-storage medium for quantum computing.

Rachel Berkowitz is a Corresponding Editor for Physics based in Vancouver, Canada.

\section{REFERENCES}

1. C. P. Moca et al., "Kondo cloud in a superconductor," Phys. Rev. Lett. 127, 186804 (2021).

Credit: C. P. Moca et al. [1] 